\title{
Is Backhaul Becoming a Bottleneck for Green Wireless Access Networks?
}

\author{
Sibel Tombaz ${ }^{1}$, Paolo Monti ${ }^{1}$, Fabricio Farias ${ }^{1,2}$, Matteo Fiorani ${ }^{1,3}$, Lena Wosinska ${ }^{1}$, and Jens Zander ${ }^{1}$ \\ ${ }^{1}$ KTH Royal Institute of Technology, Communication Systems Department, Electrum 229, 16440 Stockholm, Sweden \\ ${ }^{2}$ Federal University of Pará, Laboratory of Advanced Electromagnetism, Belém, Brazil \\ ${ }^{3}$ University of Modena and Reggio Emilia, Department of Engineering "Enzo Ferrari”, 41125 Modena, Italy \\ Email: sibel@kth.se,pmonti@kth.se, fabriciosf@ufpa.br, matteo.fiorani@unimore.it,wosinska@kth.se, jenz@kth.se
}

\begin{abstract}
Mobile operators are facing an exponential traffic growth due to the proliferation of portable devices that require a high-capacity connectivity. This, in turn, leads to a tremendous increase of the energy consumption of wireless access networks. A promising solution to this problem is the concept of heterogeneous networks, which is based on the dense deployment of low-cost and low-power base stations, in addition to the traditional macro cells. However, in such a scenario the energy consumed by the backhaul, which aggregates the traffic from each base station towards the metro/core segment, becomes significant and may limit the advantages of heterogeneous network deployments. This paper aims at assessing the impact of backhaul on the energy consumption of wireless access networks, taking into consideration different data traffic requirements (i.e., from todays to 2020 traffic levels). Three backhaul architectures combining different technologies (i.e., copper, fiber, and microwave) are considered. Results show that backhaul can amount to up to $50 \%$ of the power consumption of a wireless access network. On the other hand, hybrid backhaul architectures that combines fiber and microwave performs relatively well in scenarios where the wireless network is characterized by a high small-base-stations penetration rate.
\end{abstract}

Index Terms-Energy Efficiency, Backhaul, Fiber, Microwave, VDSL2, Power Consumption, Traffic Model, Smallcells, Heterogeneous Networks.

\section{INTRODUCTION}

Wireless access networks have undergone tremendous improvements to be able to provide high-capacity connectivity to an increasing number of mobile users. As a result, and also due to the widespread request for an (almost) ubiquitous access to data-traffic-demanding services (e.g., video), forecasts indicate that current traffic volumes will increase by 1000 times by 2020 [1]. In such a scenario operators are likely to face decreasing revenues in terms of per-unit-of-data consumed, thus highlighting the importance of having cost effective solutions in place while deploying and operating their wireless access networks. In this regard, energy efficiency is of great interest due to the fact that power consumption represents a non negligible portion of an operational expenditure (OPEX), a portion that is expected to increase even further if nothing would be done to address this issue [2].

One important step towards implementing energy efficient solutions in the wireless access network is the ability to precisely characterize the power consumption of each segment, i.e., wireless and backhaul. Even though accurate models for different base station (BS) types have been proposed (e.g., within the FP7 EARTH project [3]), relatively scarce attention has been paid to understand the role played by the backhaul in the overall network power consumption assessment. On the other hand, recent studies [4], [5] highlighted that the backhaul has a non negligible impact on the overall power budget of mobile heterogeneous networks. This is mainly due to the fact that in some cases power consumption of backhauling operations at one small BS might be comparable to the amount of power necessary to operate the BS itself [4]. Therefore, with a potential evolution towards denser heterogeneous wireless network deployments (i.e., where a massive number of small base stations are expected to be used) the power consumption of backhaul might potentially become a serious bottleneck.

The aim of this paper is to investigate the aforementioned specific aspects. With this objective in mind, we consider three different backhaul architectures and their respective power consumption models. Two architectures are based on a hybrid solution (i.e., one combining fiber and copper (VDSL-2 based) technologies, the other combining fiber and microwave) while the third backhaul architecture considered in the paper uses microwave only. In terms of wireless deployment strategy the paper presents three heterogenous deployment use cases. One is based on recent (i.e., year 2010) data traffic levels, while the other originates from the data traffic estimations for the year 2015 and 2020, respectively. These deployment scenarios are a combination of macro base stations and indoor small cells (i.e., femto base stations), an option which have attracted great interest in order to address coverage and capacity needs in residential and enterprise environments [6], [7]. The methodology followed in the study works as follows: for each specific wireless network deployment use case, the three backhaul network architectures under exam are dimensioned and their total power consumption is computed using the proposed power consumption models. The intention is to demonstrate how different assumptions on femto base station densities (used for indoor offloading), backhaul architectures, and area capacity requirements affect the total power consumption of the entire wireless access segment, while answering the questions on whether or not backhaul will be a bottleneck for future green and ultra-high capacity wireless access network deployments.

The remainder of this paper is organized as follows: Section II presents the network model and the assessment methodology. Long-term, large-scale traffic models and wireless network dimensioning are introduced in Section III. Detailed power consumption models for the selected backhaul archi- 
tectures is presented in Section IV, and numerical results are provided in Section V. The last section concludes the paper.

\section{Network Model And Methodology}

This section presents first the assumptions used and the general scenario under consideration. Then it explains the methodology used to estimate the total network power consumption.

\section{A. Network Model and Assumptions}

Let $\mathcal{A}\left[\mathrm{km}^{2}\right]$ be the dimension of the area under exam and $\rho$ (users $/ \mathrm{km}^{2}$ ) be its population density. It is assumed to have $N_{b}$ buildings each one with five floors, where each building is assumed to have ten apartments, i.e., the number of apartments $N_{a}=10 \times N_{b}$. Users are assumed to be served by a macro plus femto base station deployment (Fig. 1) where the femto BSs, placed inside apartments and operating in licensed spectrum, address the indoor coverage and capacity needs in residential/enterprise environments, while macro BSs provide an umbrella coverage and serve the remaining users in the network [7]. The considered average data rate per user changes over the years (Section III.A), while the total power consumption of each network deployment instance (function of the specific backhaul architecture under exam) is computed following the methodology described in the next sub-section.

\section{B. Methodology}

The objective of the paper is to assess the fraction of the overall power consumed by the backhaul segment within a heterogenous wireless network deployment when current and future data traffic levels are considered. The methodology of this process can be summarized as follows.

The first step is the Traffic Forecast phase. This step generates an estimate of the average area traffic demand for a dense urban area at the busy hour for the specific year under exam (i.e., 2010, 2015, and 2020). This traffic estimate is based on long-term, large scale-traffic models and on forecasted data for network and service usage such as: $(i) \rho,(i i)$ the percentage of users that are active at busy hour, (iii) their behavior (i.e., heavy vs. ordinary type), and (iv) the penetration rate of different terminal types (i.e., pc, tablet, and smart-phone). All the details regarding this phase are provided in Section III.A.

The second step consists in the Wireless Network Dimensioning phase. The result of this step is a function of the traffic forecast generated in the first phase and of a number of topology related parameters (e.g., $\mathcal{A}, \rho, N_{b}, N_{a}$, etc.,). Based on these input parameters this step returns the dimensioning for the wireless access segment, i.e., number of macro and femto BSs in addition with their peak traffic values. These numbers vary also depending on the femto base station penetration rate $(\eta)$, i.e., the fraction of apartments assumed to be equipped with femto BS. The higher is this value the lower is the number of macro base stations that will need to be deployed. It is assumed that when $\eta>0$, each building has at least one apartment with a femto BS. All the details regarding this phase are provided in Section III.B.

The third step is the Backhaul Network Dimensioning phase and provides the number of microwave antenna, fiber switches,

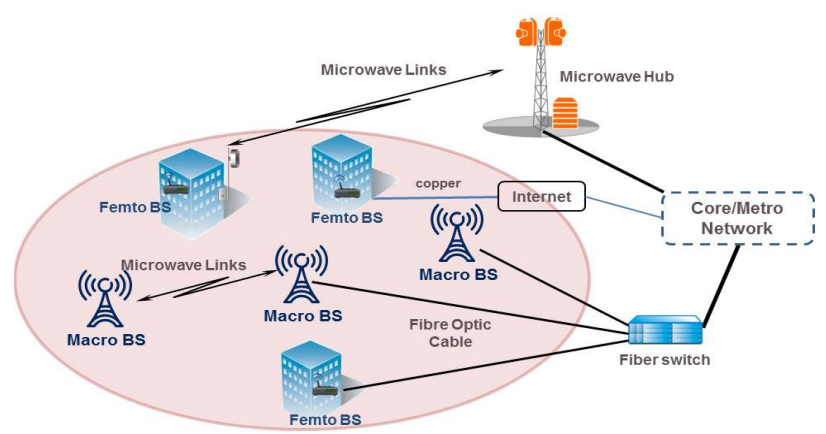

Fig. 1. Network Layout.

DSL Access Multiplexers (DSLAMs), etc. The result is a function of the outcome from Wireless Network Dimensioning phase and also of the chosen backhaul architecture. All the details regarding this phase are provided in Section IV.

Finally in the last step (i.e., Assessment of Total Power Consumption) the total power consumption of the overall wireless access network considering both the wireless and the backhaul segment is computed. The calculations are based on the power consumption models presented in Section IV.

\section{Wireless NeTWORK Dimensioning}

This section first introduces the long-term, large-scale traffic model used during the Traffic Forecast phase. Then it explains in detail how the work in the Wireless Network Dimensioning phase is carried out.

\section{A. Long-Term Large-Scale Traffic Model}

We consider the long term large scale traffic model presented in [8] in order to estimate the area traffic demand in an average European dense urban city for a given year. Since data volumes per subscriber do not depend on the specific deployment scenario, the daily generated traffic $R(t)$ over a given area can be defined as a function of $\rho$ as follows:

$$
R(t)=\rho \alpha(t) \sum_{k} r_{k} s_{k}, \quad\left[\mathrm{Mbps} / \mathrm{km}^{2}\right] .
$$

Here $r_{k}$ and $s_{k}$ represent the average data rate and the fraction of the subscribers using terminal type $k$, respectively. $\alpha(t)$ represents a typical daily traffic variation in terms of percentage of number of active users for a given time $t$. Please note that, unlike in [8], we ignore the fact that total traffic is served by a few operators in a given area.

As in [8], three different terminal types are considered: PC, tablet and smartphone. On average a PC user is assumed to generate two and eight times more data traffic than a tablet and a smartphone user, respectively [8]. On the other hand, users are divided into two groups (i.e., heavy and ordinary users) where the capacity requirement of an ordinary user is $1 / 8$ of the one of a heavy user [8]. Under the assumption that $h \%$ of the subscribers are classified as heavy users, the average daily data rate demand for terminal $k$ can be defined as:

$$
r_{k}=\left[h r_{k}^{\text {heavy }}+(100-h) r_{k}^{\text {ordinary }}\right] / 100 \quad[\mathrm{Mbps}] .
$$


Here $r_{k}^{\text {heavy }}[\mathrm{MB} /$ hour $]$ and $r_{k}^{\text {ordinary }}[\mathrm{MB} /$ hour $]$ represent the hourly average data rate of a heavy and an ordinary user, respectively.

Using (1) in combination with the forecasted values of $h$, the fraction of the subscribers using the three terminal types (i.e., $s_{p c}, s_{\text {tablet }}, s_{\text {s.phone }}$ ), and the average data rate requirements for a heavy user $r_{k}^{\text {heavy }}$ [1], [8], [9], it is possible to calculate the peak area traffic demand at the busy hour as $\mathcal{T}\left[\mathrm{Mbps} / \mathrm{km}^{2}\right]=\max _{t}(R(t))$. Table I present this value specifically for year 2010, 2015 and 2020 under the assumption that $16 \%$ of the subscribers are active during the busy/peak hour (i.e., $\alpha_{\max }=16 \%$ ), whereas $\rho=3000$ users $/ \mathrm{km}^{2}$ [8]. It should be noted that $\rho$ and $\alpha(t)$ are assumed to be constant (i.e., they do not change with time).

TABLE I

ESTIMATED AREA TRAFFIC DEMAND IN A DENSE URBAN AREA IN EUROPE FOR 2010-2020 [1], [8], [9]

\begin{tabular}{cccccc}
\hline \hline \multirow{2}{*}{ Year } & $h$ & $s_{\text {pc }} / r_{\text {pc }}^{\text {heavy }}$ & $s_{\text {tablet }} / r_{\text {tablet }}^{\text {heavy }}$ & $s_{\text {s.phone }} / r_{\text {s.phone }}^{\text {heavy }}$ & $\mathcal{T}$ \\
\hline 2010 & 10 & $0.1 / 56.25$ & $0.03 / 28.1$ & $0.3 / 7$ & 2.6 \\
2015 & 20 & $0.2 / 900$ & $0.05 / 450$ & $0.5 / 112.5$ & 82.8 \\
2020 & 30 & $0.3 / 2700$ & $0.1 / 1350$ & $0.6 / 337$ & 474.3 \\
\hline
\end{tabular}

\section{B. Macro+Femto Deployment}

For the wireless network dimensioning, it is assumed that residential femto BSs are randomly deployed by the end user in their apartments. The number of deployed femto BSs $\left(N_{\text {femto }}\right)$ is calculated as a function of the femto BS penetration rate $(\eta)$ and the total number of apartments in the area: $N_{\text {femto }}=N_{a} \times \eta$. Since the macro-cellular network needs to serve the remaining active users (i.e., which are not covered by femto BSs) at the busy hour, the required number of macro BSs in a given network area $\mathcal{A}$ can be computed as:

$$
N_{\text {macro }}=\frac{\rho \times \mathcal{A} \times(1-\eta) \times \alpha_{\max }}{N_{\text {active } / \text { macro }}} .
$$

Here $N_{\text {active/macro }}$ denotes the number of active users that can be served by a macro BS and is given by

$$
N_{\text {active/macro }}=\frac{C_{\text {macro }}}{\bar{r}}
$$

where $C_{\text {macro }}$ and $\bar{r}=\sum_{k} r_{k} s_{k}$ represent the macro BS capacity, and the average data rate requirement per active user, respectively. The fact that macro BSs can in theory support more users after offloading some indoor users to femto BSs is not considered here. It is also important to note that the fraction of the users that are served by femto BSs is assumed to be equal to $\eta$ despite the fact that more users can be within the coverage area of a femto BS, in the case open access femto BSs [7] are used.

Based on the assumption that co-channel femto BS deployment has only a minor impact on the macro BS downlink performance [6], [10], the average macro BS capacity can be calculated via the following fluid model [11]:
$C_{\text {macro }}=N_{s} W \log _{2}\left(1+\mathbb{E}_{d}\left[\frac{3 \sqrt{3}(\gamma-2)}{4 \pi} \frac{R^{2}(2 R-d)^{2-\gamma}}{d^{\gamma}}\right]\right)$

where $\gamma, N_{s}, W, R$ and $d$ denote the path loss exponent, the number of sectors, the system bandwidth, the cell radius and the distance of a user from the serving macro BS, respectively.

After using Eq. (5) to compute the average macro cell capacity over different values of $R$, results confirm that, in the case of an interference limited systems, the spectral efficiency of a base station is independent from the total number of base stations in the considered area. This results are in accordance with the earlier findings in [12]. Thus, for the scenario considered in this paper the macro BS capacity is constant regardless of number of femto and macro BS density in the area.

\section{Power Consumption Models}

The total power consumption of a heterogeneous mobile network can be defined as:

$$
\mathcal{P}=\sum_{i=1}^{m} N_{i} P_{i}+P_{b h}^{a r c h l},
$$

where $m$ is the number of base station types used in the network, $N_{i}$ and $P_{i}$ are respectively the number and the power consumption of the base stations of a specific type, and $P_{b h}^{a r c h l}$ is the power consumption of the $l$-th backhaul architecture where $l \in\{1,2,3\}$. In this paper, two BS types are considered, i.e., macro BS and femto BS, with $m=2$. The power consumption of a base stations $\left(P_{i}\right)$ is computed according to [3] and using the following equation:

$$
P_{i}= \begin{cases}N_{s}\left(a_{M} P_{t x}+b_{M}\right) & \text { for a macro BS } \\ a_{F} P_{t x}+b_{F}, & \text { for a femto BS, }\end{cases}
$$

where $P_{t x}$ denotes the power fed to the antenna. In Eq. (7), $a_{M}$ and $a_{F}$ represent the portion of the power consumption for macro and femto BS respectively, that depends on: the transmit power, the feeder losses, and the power amplifier. Finally, $b_{M}$ and $b_{F}$ represent the power consumption of the active site cooling and the signal processing for macro and femto BS respectively, and constitute the major part of the total power consumption of a BS. The backhaul power consumption models for the selected architectures will be introduced in the following subsections.

\section{A. Architecture 1: Fiber-to-the-node (FTTN) using VDSL2}

The first backhaul solution is presented in Fig. 2(a) and is given by a hybrid architecture that employs both fiber and copper. Here, femto BSs are backhauled using Very-highspeed Digital Subscriber Line version 2 (VDSL2) modems, which provide high-speed connections over copper pair cables. Each femto BS is connected to a VDSL2 modem that is in turn connected to a DSLAM using a high speed connection over copper. The DSLAM is located in a remote node that is usually placed inside a street cabinet close to the user premises. We considered VDSL2 instead of the more widespread Asynchronous DSL (ADSL) because ADSL technologies support 


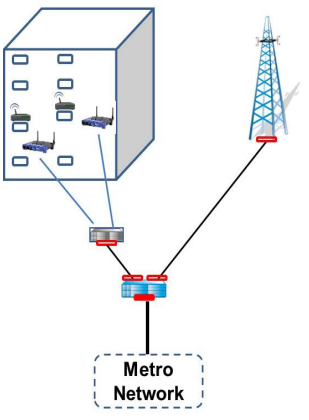

(a) FTTN using VDSL2

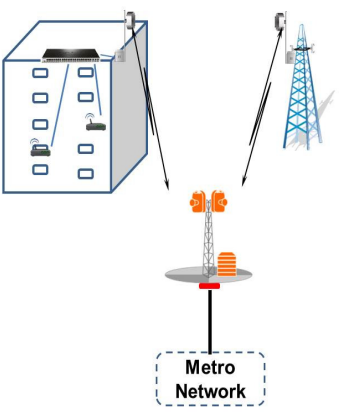

(b) Microwave only
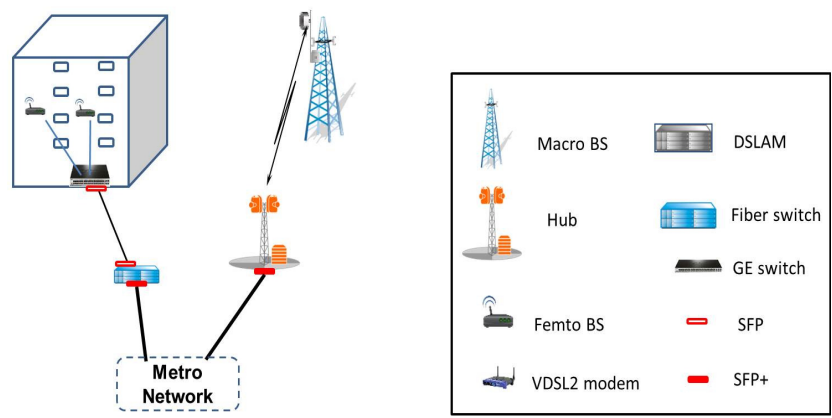

(c) FTTB + Microwave

Fig. 2. Backhaul Architectures.

only capacities in the range between 8 and $24 \mathrm{Mbps}$, which is not sufficient to backhaul a femto BS. VDLS2 provides instead $100 \mathrm{Mbps}$ for distances up to $300 \mathrm{~m}$ [13], but requires a careful network planning in order to make sure that the distance between the DSLAMs and the VDSL2 modems is less than $300 \mathrm{~m}$. DSLAMs and macro BSs are connected to a number of fiber switches (FSs) using $1 \mathrm{Gbps}$ point-to-point optical links. For transmitting and receiving the optical signal small form-factor pluggable transceivers (SFPs) are used. The FSs aggregate the traffic coming from the wireless network before sending it towards the metro network $(\mathrm{MN})$ via 10 Gbps fiber links and SFP+ modules. The power consumption of the first architecture, i.e., $P_{b h}^{a r c h 1}$, is obtained through the following formula:

$$
\begin{aligned}
P_{b h}^{a r c h 1} & =N_{\text {femto }} P_{\text {modem }}+N_{D S L A M}\left(P_{D S L A M}+2 P_{S F P}\right) \\
& +N_{s}^{F} P_{s}^{F}+2 N_{\text {macro }} P_{S F P}+N_{u l} P_{S F P+},
\end{aligned}
$$

where $P_{\text {modem }}, P_{D S L A M}, P_{s}^{F}$, and $P_{S F P}$ are power consumption values of a VDSL2 modem, a DSLAM, a fiber switch, and a SFP, respectively. On the other hand $N_{D S L A M}$ and $N_{s}^{F}$ are the number of DSLAMs and fiber switches in the area. $N_{D S L A M}$ is a function of the number of ports per DSLAM $\left(n_{\text {ports }}^{D}\right)$, i.e., $N_{D S L A M}=\left\lceil\frac{N_{\text {femto }}}{n_{\text {ports }}^{D}}\right\rceil$. Similarly, $N_{s}^{F}$ is dependent on the number of ports of a FS $\left(n_{\text {ports }}^{F}\right)$, i.e., $N_{s}^{F}=\left\lceil\frac{N_{D S L A M}+N_{\text {macro }}}{n_{\text {ports }}^{F}}\right\rceil$. Finally, $N_{u l}$ is the total number of uplink interfaces used to connect toward the $\mathrm{MN}$, while $P_{S F P+}$ is the power consumption of a SFP+ used to transmit the backhauled traffic toward the MN. $N_{u l}$ depends on the total aggregate traffic collected at the FSs, i.e., $A g_{\text {tot }}=\mathcal{T} \times \mathcal{A}$, and on the maximum transmission rate of an uplink interface $\left(U_{\max }\right) . N_{u l}$ can be computed as $N_{u l}=\max \left(N_{s}^{F},\left\lceil\frac{A g_{t o t}}{U_{\max }}\right\rceil\right)$.

\section{B. Architecture 2: Microwave Only}

The second backhaul architecture is shown in Fig. 2(b) and is based on microwave. The femto BSs inside a building are connected to a Gigabit Ethernet Switch (GES) using conventional Fast Ethernet connections operating at $100 \mathrm{Mbps}$. The GES aggregates the traffic from the femto BSs inside a building before sending it to a microwave antenna placed on the roof. Each building and each macro BS in the analyzed area is equipped with a microwave antenna operating in the frequency range between 5 and $8 \mathrm{GHz}$, which is the most suited for dense urban areas [14]. Microwave antennas are connected to hubs using a point-to-point star topology. The hubs are equipped with switches to aggregate the traffic from the microwave antennas and to transmit it toward the MN. The transmission toward the MN is realized using a $10 \mathrm{Gbps}$ optical point-to-point links and SFP+ modules. The power consumption of the second architecture, i.e., $P_{b h}^{a r c h 2}$, can be expressed as:

$$
P_{b h}^{a r c h 2}=\sum_{j=1}^{N_{b}+N_{\text {macro }}+N_{h u b}} P_{j}^{M W}+N_{G E S} P_{G E S}+N_{u l} P_{S F P+} .
$$

Here $N_{b}, N_{\text {macro }}$ and $N_{h u b}$ are the number of buildings, macro BSs, and hubs, respectively. On the other hand, $P_{j}^{M W}$ represents the power associated with MW backhaul operations at site $j$ (building, macro BS, or hub). Finally, $N_{G E S}$ is the number of GESs, and $P_{G E S}$ is the power consumption of a GES.

It can be observed that $N_{G E S}$ is equal to the number of buildings if $\eta>0$, due to the initial assumption that each building has at least one femto BS. It is also assumed that $P_{G E S}$ is a function only of the number of femto BSs in the building. Finally, the power consumption of a GES is assumed to scale linearly with the number of ports that are used for backhauling the femto BSs: $P_{G E S}=\frac{N_{\text {femto }}}{N_{b} n_{\text {ports }}^{\text {GES }}} P_{G E S}^{\max }, \forall \eta>$ 0 , where $n_{\text {ports }}^{G E S}$ and $P_{G E S}^{\max }$ are the total number of ports of the GES and its maximum power consumption, respectively. According to [5], $P_{j}^{M W}$ can be defined as:

$$
P_{j}^{M W}= \begin{cases}P_{\text {low }-c} & \text { if } N_{j}^{\text {ant }}=1 \\ P_{\text {high-c }}+P_{s}^{M W}\left\lceil\frac{C_{j}}{C_{\text {switch }}^{M W}}\right\rceil & \text { otherwise }\end{cases}
$$

where $N_{j}^{a n t}$ is the number of microwave antennas at site $j$, $C_{j}$ is the aggregated traffic at the same site, while $C_{\text {switch }}^{M W}$ 
and $P_{s}^{M W}$ are the maximum capacity of a switch inside a hub and its power consumption. Finally, $P_{l o w-c}$ and $P_{h i g h-c}$ represent respectively the low and the high power consumption region of the microwave antennas [5]. Note that $P_{j}^{M W}$ is a function of the number of antennas and not of the total backhauled capacity as it was the case in [5]. This is because sites with only one microwave antenna are assumed to work in low capacity traffic conditions (i.e., their aggregate capacity is $<500 \mathrm{Mbps}$ ), whereas the sites with more than one antennas are assumed to operate in the high traffic capacity region (i.e., their aggregate capacity is $\geq 500 \mathrm{Mbps}$ ). According to this rationale, it is assumed that each building and each macro BS is equipped only with a single antenna. Instead, the hubs, which aggregate the traffic coming from several buildings or macro BSs, are equipped with more antennas and will thus require a switch. If $\eta>0$ each building is assumed to be equipped with one GES, so that the number of GES is equal to the number of buildings (i.e., $N_{G E S}=N_{b}$ ). This is because this work assumes that the GES has enough ports to potentially connect all apartments in a building. The hubs are assumed to equally share the traffic, making the total number of hubs in the area equal to $N_{h u b}=\left\lceil\frac{N_{b}+N_{\operatorname{macro}}}{n_{s u p}^{M W}}\right\rceil$, where $n_{\text {sup }}^{M W}$ is the max number of microwave links a hub can support. With the above mentioned assumptions, Eq. (9) can be simplified to:

$$
\begin{aligned}
P_{b h}^{a r c h 2} & =\left(N_{b}+N_{\text {macro }}\right) P_{\text {low }-c}+N_{b} P_{G E S} \\
& +N_{\text {hub }} P_{\text {high }-c}+N_{u l} P_{S F P+}+N_{s}^{M W} P_{s}^{M W},
\end{aligned}
$$

where $N_{s}^{M W}$ is the total number of switches inside the hubs, i.e., $N_{s}^{M W}=\max \left(N_{h u b},\left\lceil\frac{A g_{t o t}}{C_{s w i t c h}^{M W}}\right\rceil\right)$. Finally, the total number of interfaces for the interconnection toward the MN is computed as $N_{u l}=\max \left(N_{h u b},\left\lceil\frac{A g_{t o t}}{U_{\max }}\right\rceil\right)$.

\section{Architecture 3: Fiber-to-the-Building $(F T T B)+$ Microwave}

The third backhaul solution is shown in Fig. 2(c). It is a hybrid architecture that employs both fiber and microwave. Similarly to Architecture 2, the femto BSs inside a building are connected to a GES using Fast Ethernet connections. The GES connects to a FS using 1 Gbps optical point-to-point links. SFP transceivers are used at the GES and at the FS to transmit and receive the optical signal. The FSs are connected to the MN using $10 \mathrm{Gbps}$ optical links and SFP+ transceivers. On the other hand, the macro BSs are backhauled using microwave. Again, we consider a point-to-point star topology where several microwave antennas are directly connected to a hub. The hubs are equipped with switches to aggregate the traffic from the macro BSs and are connected to the MN using $10 \mathrm{Gbps}$ optical links and SFP+ modules. Considering the same assumptions used for Architecture 2, the power consumption of Architecture 3 can be defined as:

$$
\begin{aligned}
P_{b h}^{a r c h 3} & =N_{b}\left(P_{G E S}+2 P_{S F P}\right)+N_{\text {macro }} P_{l o w-c}+N_{s}^{F} P_{s}^{F} \\
& +N_{\text {hub }}\left(P_{\text {high }-c}+P_{S F P+}\right)+N_{s}^{M W} P_{s}^{M W}+N_{u l} P_{S F P+} .
\end{aligned}
$$

It should be noted that in the architecture of Fig. 2(c) there are two types of aggregation points, i.e., (i) microwave hubs (supporting at most $n_{\text {sup }}^{M W}$ microwave links each), and (ii) fiber switches (with $n_{\text {ports }}^{F}$ each). Due to the fact that only macro BSs use microwave backhauling, the total number of hubs required in this architecture can be computed as $N_{\text {hub }}=\left\lceil\frac{N_{m a c r o}}{n_{s u p}^{M U}}\right\rceil$. On the other hand, $N_{s}^{F}=\left\lceil\frac{N_{b}}{n_{\text {ports }}^{F}}\right\rceil$. The total number of switches inside the hubs are calculated as a function of the aggregated outdoor traffic only $A g_{\text {tot }}^{\text {outdoor }}$, i.e., $N_{s}^{M W}=\max \left(N_{h u b},\left\lceil\frac{A g_{\text {tot }}^{\text {out door }}}{C_{s w \text { Witch }}^{M W}}\right\rceil\right)$. On the other hand, the number of uplink interfaces $\left(N_{u l}\right)$ are calculated based on the total aggregated traffic collected at the fiber switches and hubs, i.e., $N_{u l}=\max \left(N_{h u b}+N_{s}^{F},\left\lceil\frac{A g_{t o t}}{U_{\max }}\right\rceil\right)$.

\section{Numerical RESUlts}

In this section, we present the numerical results assessing the impact of the backhaul power consumption in future high data rate wireless access networks. We consider various realistic backhaul architectures that cope with the expected traffic demand up to 2020.

We consider a $10 \mathrm{~km} \times 10 \mathrm{~km}$ dense urban area of an average European city. The following assumptions are made: a population density of 3000 users $/ \mathrm{km}^{2} ; 100,000$ apartments; 10,000 residential buildings; the average data rate demand increases exponentially. In order to calculate the average traffic demand at busy hours, we use the forecasted values in [1], [8] together with the long- term, large-scale traffic models presented in Section III. We assume that user demand is satisfied by a macro+femto deployment strategy where the femto BS penetration rate $(\eta)$ varies between 0 and 0.6. This assures that the maximum percentage of users served by femto BSs is $60 \%$, which corresponds to the expected fraction of traffic that will be generated indoors by 2015 [7]. In this scenario, the mobile data traffic is assumed to be aggregated towards the metro network via the three candidate backhaul architectures presented in Section IV. Detailed system and power consumption parameters are listed in Table II.

TABLE II

Simulation Assumptions [4], [5]

\begin{tabular}{ll}
\hline \hline Considered parameters for wireless deployment & Value \\
\hline Population density per $\mathrm{km}^{2}$ & 3000 \\
Covered Area & $10 \mathrm{~km} \times 10 \mathrm{~km}$ \\
Number of apartments & 100000 \\
Number of buildings & 10000 \\
Bandwidth & $10 \mathrm{MHz}$ \\
Number of sector Macro/Femto & $3 / 1 \mathrm{~m}$ \\
Femto BS penetration rate & {$[0,0.6]$} \\
Path loss exponent & 3.5 \\
\hline Power Consumption Parameters & Value \\
\hline$a_{M} / a_{F}$ & $4.7 / 8$ \\
$b_{M} / b_{F}$ & $130 / 4.8 \mathrm{~W}$ \\
$P_{m o d e m}$ & $5 \mathrm{~W}$ \\
$P_{u l} / P_{d l} / P_{S F P}$ & $2 / 1 / 1 \mathrm{~W}$ \\
$P_{s}^{F} / P_{s}^{M W}$ & $300 / 53 \mathrm{~W}$ \\
$P_{D S L A M} / P_{G E}^{\max }$ & $85 / 50 \mathrm{~W}$ \\
$P_{l o w-c} / P_{\text {high }}$ c & $37 / 92.5 \mathrm{~W}$ \\
$n_{\text {ports }}^{D} / n_{\text {ports }}^{F} / n_{\text {ports }}^{G} / n_{\text {sup }}^{M W}$ & $16 / 24 / 12 / 16$ \\
$C_{\text {switch }}^{M W} / U_{\max }$ & $36 / 10 \mathrm{~Gb} / \mathrm{s}$ \\
\hline \hline
\end{tabular}




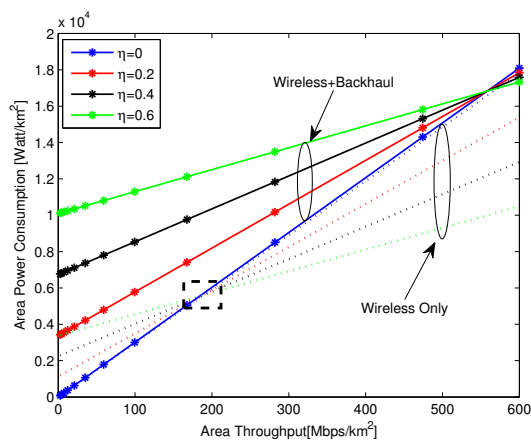

(a) FTTN using VDSL2

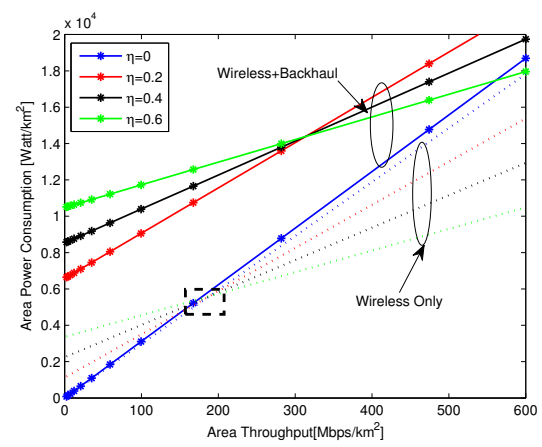

(b) Microwave only

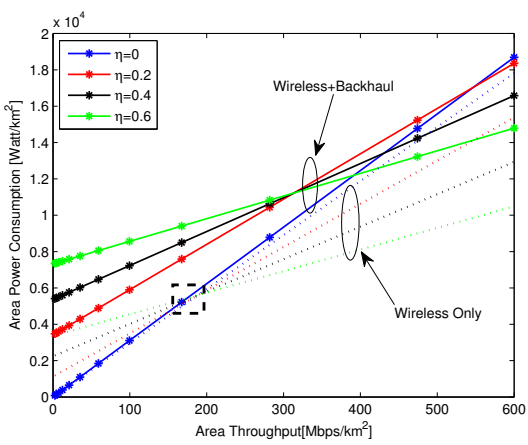

(c) FTTB+Microwave

Fig. 3. Area power consumption as a function of target area throughput considering various backhaul options.

Fig. 3 shows (for each of the three considered backhaul architectures) the area power consumption of the overall wireless access network (wireless+backhaul) as a function of the area throughput (solid lines). In order to clearly see the backhaul impact, the figure also presents the area power consumption of the wireless part only (dotted lines). Note that a macro + femto deployment can be considered as an energy efficient solution only when the additional power consumption of the large number of femto BSs is compensated by a reduction in the macro BS density. In this respect, it can be observed that if we only consider the wireless segment power consumption, a macro + femto deployment $(\eta>0)$ is more advantageous than a macroonly $(\eta=0)$ strategy only when the area throughput becomes sufficiently high, i.e., when the network is more capacity limited. Furthermore, we observe that this gain increases with denser femto BS deployment. However when also the backhaul power consumption is taken into account, the intersection point shifts to the right, i.e., much higher area capacity requirement is needed to justify a dense femto BS deployment. From Fig. 3 we observe that with a FTTN + VDSL2 and with a Microwave only backhaul solutions, the deployment of femto BSs is never beneficial for the traffic values forecasted between now and 2020 , i.e., $\mathcal{T}=474.3 \mathrm{Mbps} / \mathrm{km}^{2}$. In the case of a FTTB + Microwave backhaul solution, a dense femto BS deployment, i.e., $\eta \geq 0.4$, becomes beneficial at very high area throughput values. This clearly indicates the significant impact of backhaul power consumption on energy efficient network deployment strategies for wireless access networks.

Fig. 4 depicts a comparison between the power consumption of each presented backhaul architectures as a function of the femto BS penetration rate. In this particular experiment $\mathcal{T}=474.3 \mathrm{Mbps} / \mathrm{km}^{2}$. It is shown that among the considered backhaul solutions, FTTB + Microwave, that relies on FTTB to backhaul the indoor traffic, is the most energy efficient. On the other hand, the power consumption of FTTN using VDLS2 increases linearly with femto BS density due to the distance constraints of VDSL2 technology that leads to the deployment of very large backhaul networks. Finally the backhaul solution based on microwave only seems to be the least energy efficient due to the high consumption values of the microwave antennas that needs to be deployed at each macro BS and at each building that has at least one femto BSs. The figure also shows the clear trade-off between the power saved by using low power base stations in the wireless segment and the excess power that has to be spent to backhaul their traffic.

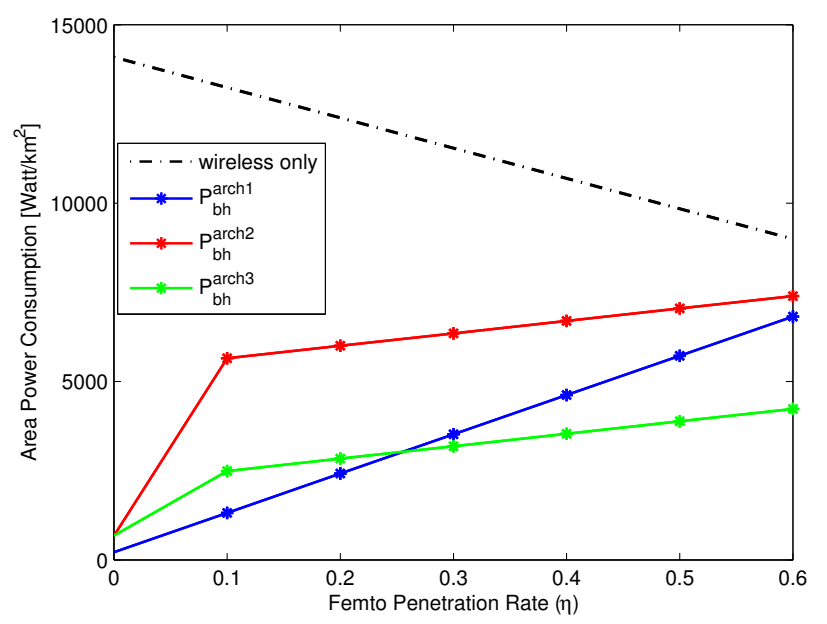

Fig. 4. Area power consumption vs. femto BS penetration rate for the traffic demand in 2020 , i.e., $\mathcal{T}=474.3 \mathrm{Mbps} / \mathrm{km}^{2}$.

Finally, the impact of backhaul on the total power consumption between 2010 and 2020 is illustrated in Fig. 5, for homogeneous, i.e., macro-only $(\eta=0)$, and heterogeneous, i.e., macro+femto $(\eta>0)$, wireless network deployment scenarios. Note that in this specific experiment it is assumed that the area traffic demand $\left(\mathcal{T} \in[2.6,474.3] \mathrm{Mbps} / \mathrm{km}^{2}\right)$ and the femto BS penetration rate $(\eta \in[0.1,0.6])$ per-year-increase is constant, i.e., $68 \%$ and $20 \%$, respectively. This was done to mimic a possible operator strategy whose goal is to offload $60 \%$ of the mobile traffic using femto BSs by 2020 . From Fig. 5 it can be observed that the area power consumption of the macro-only deployment increases exponentially due to high-densification of macro BS necessary to cope with the exponential traffic increase. However in this scenario, backhaul consume only $5 \%$ of the total power. On the other hand, even though offloading traffic to indoor femto BSs results in significant energy savings for the wireless part (Fig. 5(b)), this benefits come with a drastic increase in the backhaul power consumption, i.e., the backhaul can constitute up to $50 \%$ of 


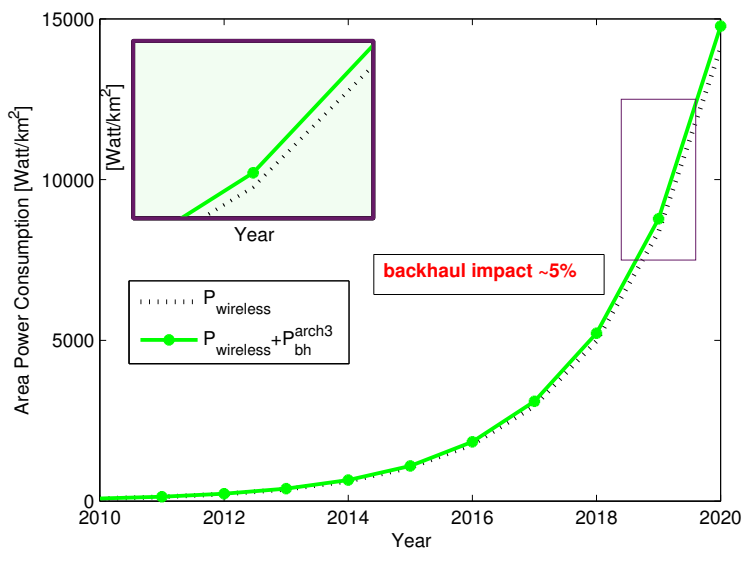

(a) Area power consumption change over the years for macro-only deployment scenario $(\eta=0)$.

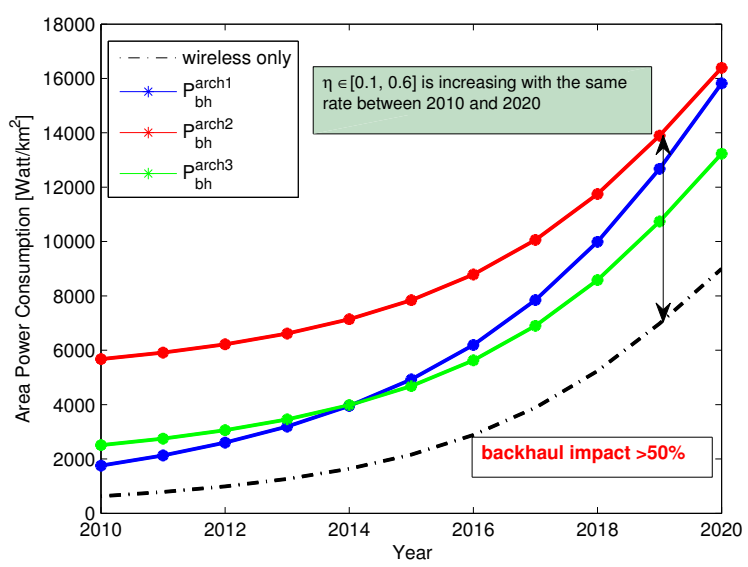

(b) Area power consumption change over the years for macro+femto deployment scenario $(\eta \in(0.1,0.6))$.

Fig. 5. Impact of backhaul on the total network power consumption over the years between 2010 and 2020. Note that mobile data traffic and femto BS penetration rate are assumed to increase respectively 68 and 20 percent annually during this period.

the total power consumption. These results clearly indicate that neglecting the backhaul power consumption while designing green wireless access network deployment strategies can result in incomplete and disputable conclusions.

\section{CONCLUSION}

In this paper, we investigated the impact of backhaul on the total power consumption of wireless access networks, taking into account the expected traffic growth between 2010 and 2020. We demonstrated how different assumptions on indoor offloading with various femto base station densities, backhaul architectures, and capacity requirements affect the total power consumption. This was done to understand whether or not backhaul will be a bottleneck in future green and ultra-high capacity wireless access networks.

The results presented in the paper indicate that backhaul can potentially become an issue in the case of dense small cells deployment. In fact, it was shown that the backhaul power consumption can amount to up to $50 \%$ of the total power consumption of the wireless access network. As a consequence, when considering a potential evolution towards heterogeneous wireless network deployments, where a massive number of small base stations will be used, backhaul power consumption has to be included in the energy efficiency analysis in order to achieve a truly green wireless access network architecture. The presented results also highlight the importance of designing energy efficient backhaul architectures. In particular, it was shown that a hybrid backhaul solution combining a Fiber to the Building (FTTB) option (i.e., to backhaul femto base stations) and microwave links (i.e., to backhaul macro base stations) is a promising candidate in scenarios where the wireless network is characterized by a high femto base stations penetration rate.

\section{ACKNOWLEDGMENT}

The work described in this paper was carried out with the support of the eWIN ("Energy-efficient wireless networking") project funded by TNG Strategic Research Area, GreenHaul ("Energy-Efficient backhauling for HetNet wireless deployments") project funded by Wireless@KTH and 5grEEn ("Towards Green 5G Mobile Network ") project funded by EIT ICT Labs.

\section{REFERENCES}

[1] "Cisco visual networking index: Global mobile data traffic forecast update, 2010-2015," February 2011.

[2] S. Tombaz, A. Västberg, and J. Zander, "Energy and cost efficient ultra high capacity wireless access," IEEE Wireless Communications Magazine, vol. 18, no. 5, pp. 18-24, Oct. 2011.

[3] G. Auer, V. Giannini, C. Desset, I. Godor, P. Skillermark, M. Olsson, M. Imran, D. Sabella, M. Gonzalez, O. Blume, and A. Fehske, "How much energy is needed to run a wireless network?" IEEE Wireless Communications Magazine, vol. 18, no. 5, pp. 40-49, Oct. 2011.

[4] S. Tombaz, P. Monti, K. Wang, A. Västberg, M. Forzati, and J. Zander, "Impact of backhauling power consumption on the deployment of heterogeneous mobile networks," in Proc. of IEEE Global Comm. Conf. (GLOBECOM), Houston, USA, Dec. 2011.

[5] P. Monti, S. Tombaz, L. Wosinska, and J. Zander, "Mobile backhaul in heterogeneous network deployment: Technology options and power consumption," in Proc. of IEEE Int. Conf. on Transparent Optical Networks (ICTON), Coventry, UK, June 2012.

[6] S. Tombaz, K. Sung, and J. Zander, "Energy efficiency assessment of wireless access networks utilizing indoor base stations," in Proc. of IEEE Personal, Indoor and Mobile Radio Commun. (PIMRC), London, UK, Sep. 2013.

[7] J. Andrews, H. Claussen, M. Dohler, S. Rangan, and M. Reed, "Femtocells: Past, present, and future," IEEE J. Sel. Areas Commun., vol. 30, no. 3, pp. 497-508, Apr. 2012.

[8] EARTH Deliverable D2.3, "Energy efficiency analysis of the reference systems, areas of improvements and target breakdown," Jan. 2012.

[9] "Mobile traffic forecasts 2010-2020, report no:44," May 2011.

[10] H. Claussen, "Co-channel operation of macro- and femtocells in a hierarchical cell structure," International Journal of Wireless Information Networks, vol. 15, no. 3-4, pp. 137-147, Dec. 2008.

[11] J. M. Kelif, M. Coupechoux, and P. Godlewski, "A fluid model for performance analysis in cellular networks," EURASIP J. Wireless Commun Networking, Jan. 2010

[12] A. Ghosh, N. Mangalvedhe, R. Ratasuk, B. Mondal, M. Cudak, E. Visotsky, T. Thomas, J. Andrews, P. Xia, H.-S. Jo, H. Dhillon, and T. Novlan, "Heterogeneous cellular networks: From theory to practice," IEEE Communications Magazine, vol. 50, no. 6, pp. 54-64, 2012.

[13] W. Vereecken, W. Van Heddeghem, M. Deruyck, B. Puype, B. Lannoo, W. Joseph, D. Colle, L. Martens, and P. Demeester, "Power consumption in telecommunication networks: overview and reduction strategies," IEEE Communications Magazine, vol. 49, no. 6, pp. 62-69, 2011.

[14] Paoloni, M., "Crucial economics for mobile data backhaul," Senza Fili Consulting, 2011. 